\title{
Estudo sobre o Viés de Itens em Testes de Rendimento: uma retrospectiva
}

\author{
WAGNER BANDEIRA ANDRIOLA \\ Professor da Faculdade de Educação da Universidade Federal do Ceará - UFC \\ Coordenador de Avaliação Institucional e Análise da \\ Pró-Reitoria de Planejamento da UFC \\ w_andriola@yahoo.com
}

\begin{abstract}
Resumo
O texto retrata a origem dos estudos sistemáticos que abordam a temática dos vieses dos itens e dos testes educacionais, ressaltando os procedimentos mais freqüentemente utilizados em tal atividade. Destaca o conceito do termo funcionamento diferencial do item (DIF), caracterizando-o como adverso ou benigno a um determinado grupo demográfico. Além do mais, enfatiza a existência do DIF uniforme ou consistente e do DIF não-uniforme ou inconsistente. Por fim, destaca a idéia central: a presença do DIF é um fator de injustiça à atividade de avaliação educacional.

Palavras-chave: testes de rendimento, funcionamento diferencial do item (DIF), avaliação educacional.
\end{abstract}

\section{Resumen}

El texto retrata el origen de los estudios sistemáticos sobre el sesgo de los ítems y de los testes educativos, destacando los procedimientos que se utilizan con más frecuencia en tal actividad. Destaca el concepto del término funcionamiento diferencial del ítem (DIF), caracterizándolo como adverso o benéfico a un determinado grupo demográfico. Además, enfatiza la existencia del DIF uniforme o consistente y del DIF no-uniforme o inconsistente. Finalmente, pone de relieve la idea central: la presencia del DIF es un factor de injusticia en la actividad de evaluación educativa.

Palabras-clave: testes de rendimiento, funcionamiento diferencial del ítem (DIF), evaluación educativa.

\begin{abstract}
This paper discusses the origin of systematic studies concerning items bias in achievement tests, emphasizing the proceedings most frequently used in such activities and emphasizes the concept of the term "differential item functioning" (DIF) characterizing it as adverse or benign to a specific demographic group. It also gives prominence to the existence of uniform or consistent DIF as well as non-uniform or inconsistent DIF. It finally emphasizes the central idea: the presence of DIF is a factor of injustice in educational evaluation.
\end{abstract}

Key words: achievement tests, differential item functioning (DIF), educational evaluation. 


\section{ORIGEM DOS ESTUDOS ACERCA DO VIÉS DOS TESTES DE RENDIMENTO}

O viés dos instrumentos de medida educacional e psicológica é um tópico que aparece tardiamente tratado no seio da psicometria moderna (Muñiz, 1994). De acordo com Angoff (1993), seu estudo sistemático iniciou-se nos Estados Unidos, no final dos anos 1960, numa época em que estavam em moda os debates acerca dos direitos civis e das desigualdades de oportunidade entre brancos e minorias étnicas.

Os resultados dos processos de avaliação educacional, executados por reconhecidas instituições, tal como o Educational Testing Service (ETS), foram discutidos por diversos intelectuais, como sociólogos e pedagogos. Segundo eles, as diferenças de rendimento, observadas entre os diversos grupos étnicos e socioeconômicos, refletiam, na realidade, disparidades nas oportunidades educacionais e discriminação contra grupos minoritários de negros, hispano-americanos, judeus e árabes (Allen, Wainer, 1989). Podemos observar, desse modo, que foi a discussão social, alheia em grande parte ao círculo psicométrico, que obrigou os especialistas da área a gerar novos procedimentos analíticos, e tinha por objetivo provar que seus testes ou instrumentos de medida não possuíam nenhum tipo de viés (Cole, 1993).

Nessa mesma época, os investigadores começaram a preocupar-se com o estudo sistemático das diferenças entre os mencionados grupos demográficos, pois estavam interessados em buscar explicações a respeito das suas verdadeiras causas explicativas. Martínez Arias (1997) destaca que a investigação acerca do viés dos itens pode remontar-se aos estudos realizados por A. Binet, em 1910, a respeito das diferenças de status socioeconômico no rendimento dos sujeitos submetidos a alguns testes desenvolvidos por ele próprio. Os resultados obtidos possibilitaram a proposição da hipótese de que o rendimento mais baixo desses sujeitos, em alguns itens, poderia ser efeito da cultura, em vez de ser fruto de potenciais diferenças na capacidade mental ou no construto latente medido pelo teste (Andriola, 2002). Também W. Stern, o introdutor do termo Quociente Intelectual, pode ser considerado um dos primeiros investigadores da área; estudou as diferenças relacionadas com a classe social, na Alemanha.

Apesar desses autores pioneiros, o começo da moderna investigação sobre o viés encontra-se nos trabalhos de K. Eells, A. Davis, R. J. Havighurst, V. E. Herrick e R. W. Tyler, realizados na Universidade de Chicago, em 1951. Nesses estudos, os citados autores encontraram variações nos itens, em alguns aspectos muito peculiares, tais como conteúdo e formato, que reduziam ou exageravam as diferenças 
observadas entre os grupos comparados (Hambleton, Swaminathan, Rogers, 1991).

Nesse contexto, surgem os primeiros dados a respeito dos problemas técnicos presentes em alguns itens dos testes então utilizados na avaliação da aprendizagem. $\mathrm{O}$ uso indevido da linguagem escrita, que possibilitava certa vantagem de um grupo de sujeitos sobre outro, era um desses problemas técnicos. Em suma: muitos dos termos empregados nos testes eram mais familiares a alguns grupos especificos de estudantes, tais como os norteamericanos brancos, originários da classe média (Linn, Harnisch, 1981). Em conseqüência, os sujeitos pertencentes aos grupos minoritários, que não conheciam ou não empregavam cotidianamente esses termos, tinham rendimento mais baixo. Surge, então, o interesse pela investigação sistemática do viés dos itens (Cole, 1993).

No âmbito da Teoria Clássica dos Testes (TCT) o termo viés é utilizado para rotular os itens que têm parâmetros de dificuldade ou de discriminação diferentes, nos distintos grupos estudados. Segundo Camilli e Shepard (1994), o viés é uma fonte de invalidez ou de erro sistemático, que se reflete em como um teste mede os membros de um grupo particular. É sistemático porque cria uma distorção nos resultados do teste, favorável ou contrário aos membros de um grupo determinado.

Faz-se mister destacar: a idéia de grupo é central nas diversas definições de viés e, por esse motivo, ele tem sido estudado, fundamentalmente, nas investigações acerca das diferenças relacionadas com algumas características grupais, como: classe social, idade, região, habitat ou outra característica sociodemográfica relevante (Andriola, 2002).

\section{PROCEDIMENTOS PARA A DETECÇÃO DO VIÉS DOS ITENS}

No estudo sistemático do viés dos itens utilizam-se duas aproximações estatísticas. Uma delas usa um critério externo ao teste, e a outra um critério interno, normalmente as pontuações ou escores totais obtidos (Whitmore, Shumacker, 1999). De acordo com Osterlind (1979, 1989), o viés externo é o grau em que as pontuações do teste têm correlações com variáveis irrelevantes para sua interpretação e alheias a este. Normalmente, ao falar do viés externo, se faz referência ao teste total e às conseqüências sociais de seu uso; o viés interno se refere às propriedades métricas dos itens dos testes. As técnicas que o detectam podem ser consideradas um tipo particular de análise de itens, que tentam responder a indagação: itens de testes padronizados têm o mesmo comportamento estatístico para diferentes subgrupos de sujeitos extraídos da mesma população? 
Para Martínez Arias (1997), o termo viés interno tem um significado preciso, único, e se considera como um erro sistemático no processo de medida; é um termo técnico, sem conotações sociais ou políticas. Os itens são considerados mais ou menos difíceis para um grupo particular, comparativamente ao rendimento de outros grupos extraídos da mesma população e com o mesmo nível de aptidão na variável latente. Os itens do teste se examinam por diferentes procedimentos ou métodos de detecção de viés. O objetivo é observar se se conforma ou não um conjunto de regras psicométricas para todas as pessoas de uma população, independentemente de que estas pertençam a grupos particulares dessa mesma população.

O viés dos itens pode ser inserido no contexto da validade de construto dos itens, isto é, o grau em que um item ou conjunto de itens mede uma mesma característica ou construto latente. No âmbito da Teoria de Resposta ao Item (TRI), a probabilidade de que um sujeito responda corretamente a um item se denomina probabilidade de êxito. Podemos estudar o viés, comparando as probabilidades de êxito para diferentes grupos da mesma população (Angoff, 1993). Desse ponto de vista, um item é considerado não enviesado se a probabilidade de êxito é a mesma para sujeitos com igual aptidão, independentemente do grupo ao qual pertença. Por outro lado, um item enviesado será aquele em que as probabilidades de êxito são diferentes, apesar da igualdade dos sujeitos na capacidade avaliada. Não obstante, se dois sujeitos têm a mesma magnitude na variável latente medida por um item qualquer, poderíamos nos perguntar: por que um item planejado para medir essa variável tem funcionamento diferencial, isto é, favorece a um grupo determinado?

Ercikan (1998) tenta responder a tal indagação, recordando-nos que na própria atividade de elaboração dos itens surgem algumas possíveis causas ou fontes de vieses, devido, sobretudo, ao:

- uso de termos conhecidos por grupos demográficos muito específicos;

- uso de termos que têm distintas significações, segundo o contexto ou características grupais dos respondentes;

- tamanho e complexidade da sentença empregada no enunciado ou nas alternativas.

Logicamente, os estudos que buscam as causas do DIF, a partir de variáveis demográficas, partem do suposto de que as fontes propostas por Ercikan (1998) não estão presentes nos itens analisados. No caso de inexistir segurança no cumprimento desse suposto, é aconselhável realizar 
algum estudo qualitativo prévio ao estudo do DIF, que esteja baseado na valoração dos itens por expertos na área (Angoff, 1993).

Muñiz (1997) tem a mesma opinião de Ercikan (1998) e Angoff (1993), já que para ele o procedimento mais eficiente para evitar o viés dos itens é por meio de uma cuidadosa análise do seu conteúdo, feita por vários expertos, antes de sua utilização definitiva. Realizada tal revisão e aplicados os itens aos sujeitos, ainda assim devem ser executadas certas análises estatísticas que permitam identificar o funcionamento diferencial naqueles itens que "escaparam" da detecção pelo uso dos procedimentos prévios.

Alguns autores, entre os quais Camilli e Shepard (1994), insistem em que os índices estatísticos empregados na análise do DIF, por si mesmos, não proporcionam prova de viés, preferindo denominá-los indices de discrepância ou de funcionamento diferencial. Historicamente, o conceito de viés sempre esteve associado ao de DIF, ainda que ambas as definições sejam distintas (Hidalgo Montesinos, López Pina, Sánchez Meca, 1997). Para Cole e Moss (1989) o viés é uma possível causa do DIF, ou seja, as diferenças observadas no funcionamento do item são provocadas por algo irrelevante ao propósito do teste.

Segundo Camilli e Shepard (1994), o DIF engloba os diferentes procedimentos estatísticos para a detecção de um possível funcionamento diferencial; insistem que este não é sinônimo de viés, ainda que alguns autores parecem crer que sim. Os métodos estatísticos de DIF serão utilizados para identificar itens que exibem funcionamento diferencial para distintos grupos. Posteriormente, depois de uma análise lógica ou experimental, no contexto da validade de construto dos itens, se determinará quais deles estão enviesados, para que sejam eliminados do teste ou do banco de itens (Andriola, 1998).

Em outras palavras, os métodos DIF são procedimentos estatísticos e as análises de viés se situam no contexto mais geral da validade de construto, ainda que neste último se usem os resultados obtidos com a aplicação do primeiro. Como assinalam Camilli e Shepard (1994) e Mellenbergh (1989), os índices DIF, às vezes, produzem resultados estatisticamente significativos na ausência de viés e não detectam o viés quando este se encontra presente em muitos itens, dada a circularidade do critério interno que utilizam. 


\section{DEFINIÇÃO DO TERMO FUNCIONAMENTO DIFERENCIAL DO ITEM (DIF)}

Com o recente surgimento do paradigma psicométrico denominado Teoria da Resposta ao Item (TRI), novas áreas de investigação têm proliferado (Andriola, 1998; Hambleton, 1989, 1990). Segundo Hambleton (1997), uma delas tem seu foco dirigido ao estudo do Funcionamento Diferencial do Item (DIF), que caracteriza um dos mais graves problemas presentes nas atividades de avaliação educacional e psicológica, pois se trata de um fenômeno observado em muitos dos itens utilizados em testes de rendimento e psicológicos.

As investigações para a detecção do DIF têm por base uma mesma argumentação: a existência do DIF é um fator que influencia a validade da interpretação, que é realizada a partir da pontuação obtida pelo sujeito num item ou teste (Andriola, 2002). Não podemos esquecer que sobre a interpretação da pontuação, seja no âmbito educativo ou psicológico, reside toda a credibilidade e reputação da investigação e da avaliação (Downing, Haladyna, 1997). Assim, está plenamente justificada a relevância das pesquisas que estudam o DIF, sobretudo aquelas que buscam identificar suas causas (Andriola, 2000).

Nesse âmbito, a necessidade e a relevância da padronização ou uniformização das condições de aplicação dos instrumentos de medida é um dos supostos mais importantes da avaliação, seja no âmbito psicológico ou educativo (Anastasi, 1988; Pasquali, 2000). Para tanto, psicólogos e pedagogos tratam de uniformizar as tarefas ou itens, as instruções, o tempo destinado à resolução das tarefas contidas nos instrumentos, a maneira de corrigir as respostas dos respondentes, as condições de luminosidade, som e a própria atividade de aplicação dos instrumentos de medida, etc. (Martínez Arias, 1997). Devemos ter claro que a presença de DIF num teste é um fator que torna o processo avaliativo injusto.

Para compreendermos essa última afirmação, devemos conhecer o conceito de DIF. É possível dizer, no âmbito da TRI, que o item não tem DIF, quando a curva característica do item (CCI) é idêntica para os grupos comparados em um mesmo nível ou magnitude da variável latente medida através do item (Lord, 1980; Mellenbergh, 1989). Em linguagem matemática poderíamos dizer que o item não tem DIF com respeito à variável $G$ (grupo) dado $Z$ (nível de $\theta$ ) se, e somente se, $F(X \mid g, z)=F(X \mid z)$, onde:

- X é a pontuação no item;

- $g$ é o valor obtido segundo a variável G;

- $z$ é o valor obtido segundo a variável $Z$. 
Nesse contexto, os valores esperados (E) são dados por $\mathrm{E}(\mathrm{X} \mid \mathrm{g}, \theta)=$ E (X $\mid \theta)$ para todo g e $\theta$. No caso de itens dicotômicos, os valores esperados são as probabilidades de acerto ao item, que podem ser expressas nos seguintes termos:

$\mathrm{P}(\mathrm{X}=1 \mid \mathrm{g}, \theta)=\mathrm{P}(\mathrm{X}=1 \mid \theta)$ para todo $\mathrm{g}$ e $\theta$. No segundo caso $[\mathrm{P}(\mathrm{X}$ $=1 \mid \theta)$, a equação expressa, na realidade, a curva característica do item $(\mathrm{CCI})$.

Geralmente, os estudos para a determinação do DIF utilizam dois grupos, denominados de referência (GR) e focal (GF). Como já enfatizamos, em termos da TRI, um item tem DIF se para valores iguais de $\theta$ não correspondem valores iguais de $\mathrm{P}(\theta)$ nas $\mathrm{CCI}^{\prime}$ s dos grupos considerados, isto é, quando $\mathrm{T}_{\mathrm{jGR}}(\theta) \neq \mathrm{T}_{\mathrm{jGF}}(\theta)$, onde:

- $T_{j G R}$ é a pontuação verdadeira do sujeito j pertencente ao grupo de referência e que possui uma certa magnitude na variável latente $\theta$;

- $\mathrm{T}_{\mathrm{jGF}}$ é a pontuação verdadeira do sujeito $\mathrm{j}$ pertencente ao grupo focal e que possui uma certa magnitude na variável latente $\theta$.

De acordo com Oshima, Raju, Flowers e Slinde (1998), a pontuação verdadeira em um teste unidimensional, composto por $k$ itens, é expressa pela fórmula:

$$
T_{S}=\sum_{i=1}^{K} P_{i}\left(\theta_{S}\right)
$$

Onde:

- $\mathrm{P}_{\mathrm{i}}\left(\theta_{\mathrm{s}}\right)$ é a probabilidade de acertar ao item $i$ pelo sujeito $s$ com a habilidade $\theta_{\mathrm{s}}$.

Segundo Mazor, Hambleton e Clauser (1998), o uso do número de respostas corretas para a determinação do DIF, isto é, a pontuação verdadeira no teste ou item, só é aceitável se o teste for unidimensional e se as respostas forem dicotômicas. a figura 1 .

Para visualizar o DIF de um hipotético item, apresentamos, a seguir, 
Figura 1 - Representação das CCI's de um Item com DIF

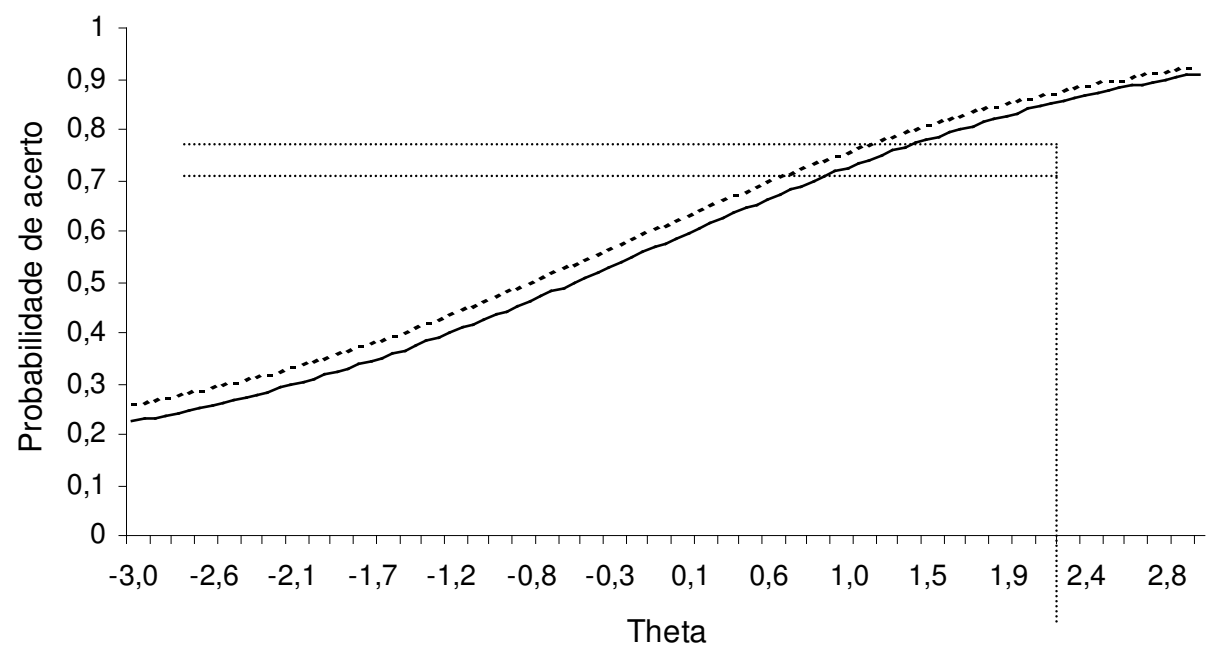

Legenda: Linha superior: CCI das mulheres; Linha inferior: CCI dos homens.

Observamos que, para uma mesma magnitude $\theta$, o valor $\mathrm{P}(\theta)$ é sempre superior para as mulheres (curva tracejada descontínua), ou seja, em níveis iguais de competência na variável medida $\theta$ não correspondem probabilidades iguais de superar o item. Neste caso, o item está enviesado contra os homens (GR) pois os valores $\mathrm{P}(\theta)$ para um mesmo nível $\theta$ são sempre maiores para as mulheres (GF). Por exemplo, para $\theta=1,4$ temos valores aproximados de $\mathrm{P}(\theta)=0,75$ para os homens e $\mathrm{P}(\theta)=0,80$ para as mulheres.

Como conseqüência de resultados dessa natureza, Douglas, Roussos e Stout (1996) propuseram os conceitos de DIF benigno e DIF adverso. No caso do DIF beneficiar o grupo de referência, isto é, quando $\mathrm{T}_{\mathrm{jGR}}(\theta)>\mathrm{T}_{\mathrm{jGF}}(\theta)$, caracteriza-se a existência de DIF benigno. O DIF adverso ocorre no caso do DIF beneficiar o grupo focal, ou seja, quando $\mathrm{T}_{\mathrm{jGR}}(\theta)<\mathrm{T}_{\mathrm{jGF}}(\theta)$. No exemplo da figura 1, temos um caso de DIF adverso. Utilizando o mesmo item, aclaremos o que ocorre na ausência de DIF, observando a figura $2 .{ }^{1}$

1 Adaptado de Andriola (2002). 
Figura 2 - Relação entre Habilidade, Item e Grupo na Ausência de DIF

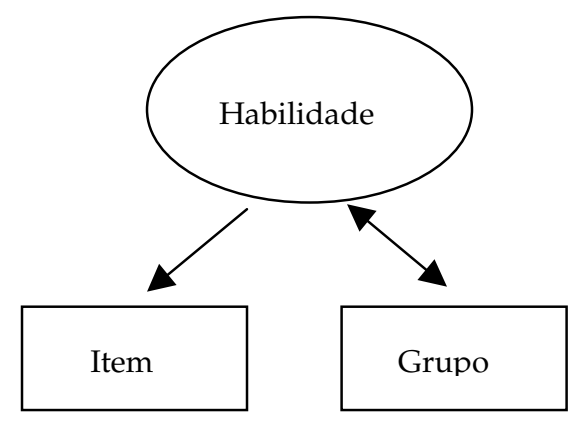

A elipse indica a habilidade ou construto latente, que tem relação causal com o item. Grupo e variável latente estão associados. Em outras palavras e a título de exemplificação, poderíamos dizer que as mulheres têm elevada habilidade na variável latente e que esta variável tem relação causal com o item, isto é, o grupo com maior capacidade na variável latente - as mulheres - tem mais respostas corretas no item. Neste caso, o rendimento no item depende, exclusivamente, da magnitude da variável latente que os indivíduos tenham, ou seja, trata-se de um item sem DIF. Agora, vejamos o que ocorre no caso de um item com DIF, observando a figura $3 .^{2}$

Figura 3 - Relação entre Habilidade, Item e Grupo na Presença de DIF

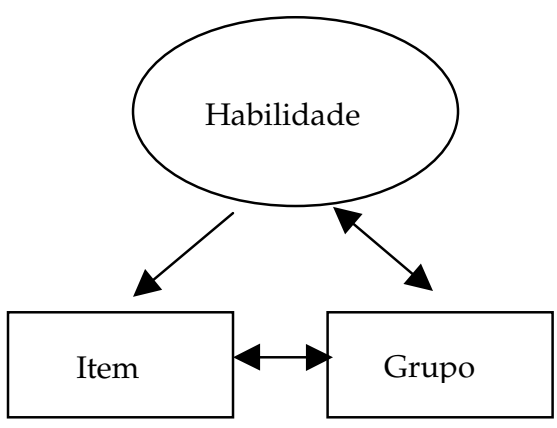

2 Adaptado de Andriola (2002). 
No caso da figura 3, temos a mesma situação descrita na figura 2, adicionada ao fato de haver associação ou interação entre grupo e item. Neste segundo caso, a associação entre ambas pode favorecer o rendimento superior de um grupo sobre o outro devido, sobretudo, a algumas características demográficas específicas tais como: gênero, raça, background educativo, origem social, etc. (Clauser, Nungester, Swaminathan, 1996). Neste caso, supõe-se que a magnitude da variável latente está sendo controlada, ou seja, os sujeitos são comparados com respeito ao seu rendimento, considerando-se que possuem a mesma aptidão. Este segundo exemplo caracteriza o caso em que o rendimento no item não depende somente da magnitude que os indivíduos tenham na variável latente, mas das características do grupo, ou seja, trata-se de um item com DIF. Em nosso exemplo, a característica do grupo que afeta o rendimento diferencial no item é o fato de o sujeito ser homem ou mulher, isto é, trata-se de uma característica de natureza demográfica, que afeta sistematicamente as respostas dos sujeitos de mesma habilidade.

Portanto, é necessário reconhecer que a presença de DIF ocasiona sérias implicações ao processo de avaliação, já que pode privilegiar um determinado grupo em detrimento de outro (Douglas, Roussos, Stout, 1996), conforme observamos no exemplo comparativo do rendimento dos homens e mulheres. Muñiz (1997) chama a atenção para o fato de que tal problema pode ter repercussões sociais mais graves se é, precisamente, a cultura dominante que elabora os itens para avaliar os demais sujeitos oriundos de outras culturas. Por exemplo, suponhamos que são construídos itens para avaliar a capacidade de raciocínio verbal em alunos de escolas públicas e privadas. Ocorre que os alunos desses tipos de escolas são, geralmente, oriundos de classes sociais distintas, com diferentes bagagens culturais, sociais, econômicas, etc. (Andriola, 1997). Todos esses aspectos contribuem para que um tipo de aluno tenha o vocabulário mais rico que o outro. Como o raciocínio verbal é medido por meio de itens que utilizam palavras, muito provavelmente, aquele tipo de aluno que conheça melhor o vocabulário utilizado nos itens terá uma clara vantagem na sua resolução (Andriola, Pasquali, 1995).

Em síntese, argumentamos que, dada a grande variabilidade de todos esses antecedentes históricos dos sujeitos implicados na avaliação do raciocínio verbal, se o item ou teste, em geral, se apóia mais nos antecedentes de uma cultura que nos de outra, terá altíssima probabilidade de não ser eqüitativo, de estar enviesado. Em outras palavras, se confunde o efeito da capacidade de raciocínio verbal (construto principal) com o conhecimento vocabular (construto secundário), isto é, se um aluno pontua baixo no teste não saberemos, ao certo, se devemos atribuí-lo à sua baixa 
capacidade de raciocínio verbal ou ao seu baixo conhecimento vocabular. Como nos fala Muñiz (1997), a casuística é interminável e pode-se dizer que não existem provas inteiramente isentas de viés. Trata-se, assim, de detectar a quantidade de viés que pode ser aceitável em um teste ou item.

Finalmente, deve ser mencionado que, nesse contexto, a importância dos estudos que objetivam a verificação do DIF está plenamente justificada. Cabe ao avaliador verificar se em seu teste existem itens com DIF, para que: 1) possa buscar as causas que o expliquem; 2) evitar sua utilização com o grupo em desvantagem; e, finalmente, 3 ) controlar os fatores responsáveis pelo DIF para evitar, desse modo, construir novos itens com o mesmo viés (Hambleton, 1989; Mislevy, 1996).

Anteriormente, destacamos o intensivo uso de testes no contexto norte-americano. Permanece, ainda hoje, a importância desses instrumentos, sobretudo na área que investiga o Funcionamento Diferencial do Item (DIF). Para termos noção dessa relevância, apresentamos parte do discurso proferido por Ree (1993), no renomado Educational Testing Service (ETS):

Nós, militares americanos, gozamos de uma posição singular porque desenvolvemos e utilizamos nossos próprios testes. Este ano, mais de 2 milhões de jovens, homens e mulheres, serão testados no processo de seleção e outras centenas de milhares de testes serão aplicados pelo serviço para efeitos de promoção e certificação. Esses testes afetarão significativamente a vida desses militares e a segurança do nosso país. [...] Para a Aeronáutica, que produz testes para alistados e oficiais, certos modelos de deteç̧ão do DIF tornaram-se parte integrante da produção de testes e dos processos de avaliação. Ademais, a validação de conteúdo e construto da grande variedade de nossos testes beneficiase das análise do DIF. (p. xi-xii)

Obviamente, o estudo do Funcionamento Diferencial do Item (DIF) também é relevante no campo da avaliação educacional, pois como destacam Hartle e Battaglia (1993):

Os baixos resultados obtidos pelas minorias e mulheres são um problema especial para as políticas públicas nacionais. A maioria dos programas nacionais são propostos para incrementar as oportunidades educativas para os grupos em desvantagem. [...] Por outro lado, também é necessário incrementar o interesse por novas técnicas de avaliação que não causem um impacto racial desproporcional. (p. 305)

Apresentamos, a seguir, os dois tipos de Funcionamento Diferencial do Item (DIF) observados no âmbito da Teoria da Resposta ao Item (TRI). 


\section{DESCRIÇÃO DOS TIPOS DE DIF NO ÂMBITO DA TRI}

Enfatizamos que, no contexto da TRI, a lógica para a detecção do DIF consiste em comparar as CCI's dos itens, considerando os grupos de referência (GR) e focal (GF), pela utilização de métodos apropriados para tal. Os distintos métodos para a detecção do DIF foram desenvolvidos com base nos vários tipos de DIF (Bock, 1993), sendo o mais conhecido denominado DIF uniforme ou consistente, que é observado quando as CCI's do item estudado para o GR e para o GF são diferentes, mas não se cruzam em nenhum ponto ao longo da magnitude da variável latente $(\theta)$, conforme representado na figura 4 .

\section{Figura 4 - Representação de um Item com DIF Uniforme}

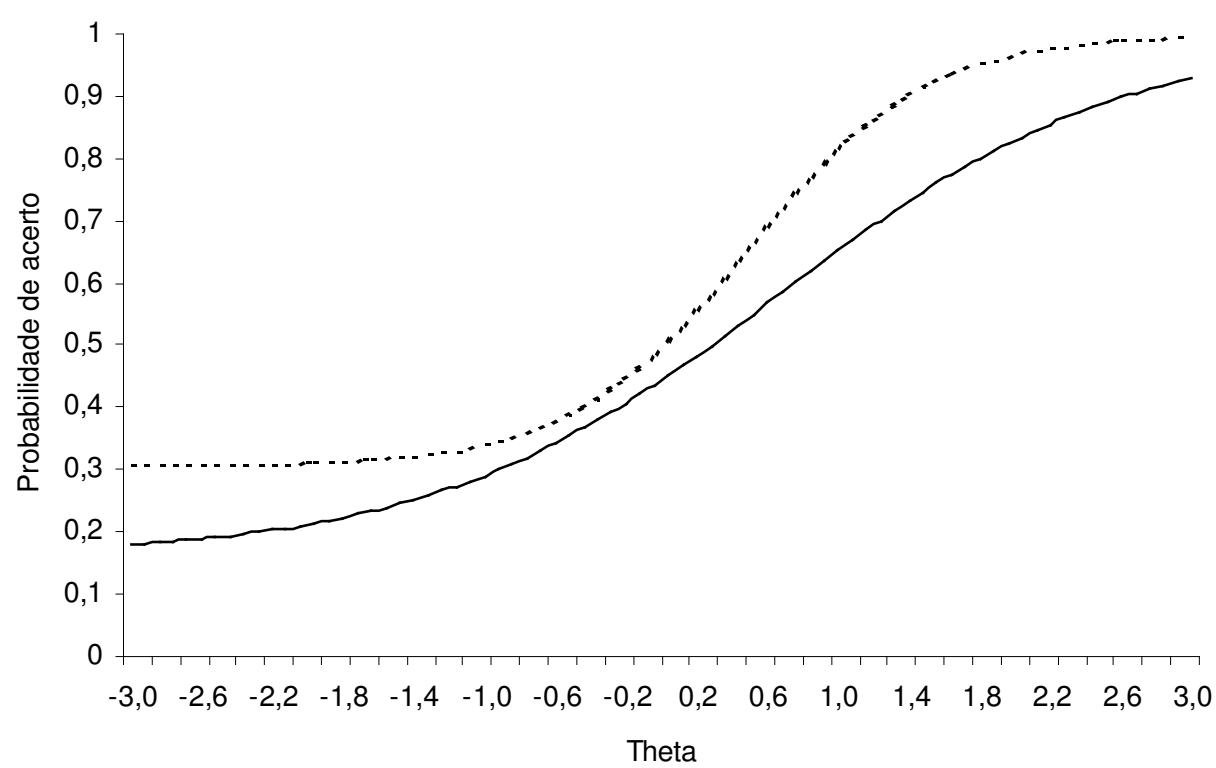

Na figura 4, observamos que a CCI do grupo focal (linha tracejada descontínua) está situada mais à esquerda que a CCI do grupo de referência (linha tracejada contínua), o que indica que o item é mais fácil para o grupo focal ao longo de toda a magnitude de $\theta$, visto que $\mathrm{P}_{\mathrm{GF}}(\theta)>$ $\mathrm{P}_{\mathrm{GR}}(\theta) \mid \theta$. Essa diferença supõe que o item tem DIF; nesse caso, benigno ao grupo de referência. 
O segundo tipo de DIF é denominado DIF não uniforme ou inconsistente, e se observa quando as CCI's do item estudado, com respeito aos grupos de referência e focal, são diferentes e, ademais, se cruzam em algum ponto do contínuo da habilidade $(\theta)$. Em outras palavras, existe uma vantagem relativa para um dos grupos investigados, cujo valor é variável durante toda a habilidade $(\theta)$, conforme representado na figura 5 .

\section{Figura 5 - Representação de um Item com DIF não Uniforme}

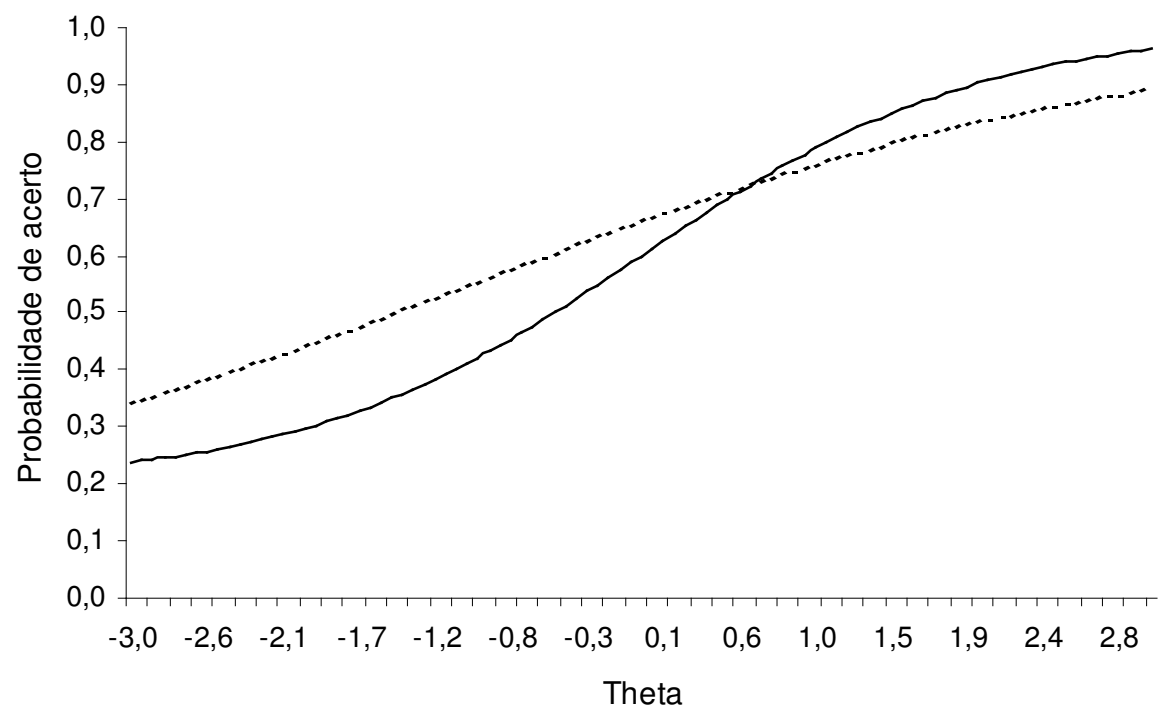

Neste segundo tipo de DIF é inapropriado examinar globalmente os dados, porque tal procedimento poderia ocultar sua presença, pois a peculiar variabilidade do DIF, que se verifica em distintas zonas da variável latente, pode cancelar, total ou parcialmente, sua detecção (Martínez Arias, 1997). É o caso de não se utilizar, por exemplo, o procedimento denominado Differential Bundles Functioning (DBF), que estuda o DIF dos itens a partir de sua organização em subconjuntos (bundles) com características comuns (Douglas, Roussos, Stout, 1996).

Autores como Cohen, Kim e Baker (1993) distinguem diferentes pesquisas sobre o DIF, segundo os objetivos pretendidos, isto é, pode-se falar de estudos para a detecção do DIF e outros para o descrição do impacto do DIF. No primeiro grupo, estão as investigações que empregam algum método tradicional para a identificação do DIF. Nesse caso, os estudos 
objetivam somente detectar o DIF, ou seja, determinar a possível diferença entre as curvas características dos itens (CCI's) de acordo com os grupos comparados. No segundo grupo, estão as investigações realizadas tendo por objetivo identificar as causas do DIF. Nesse âmbito, segundo Clauser, Nungester e Swaminathan (1996), o objetivo do investigador, que utiliza as distintas técnicas para a detecção do DIF, é tentar saber quais são as razões (psicológicas, educacionais, culturais, sociais, atitudinais, etc.) que, teoricamente, acarretam o funcionamento diferencial do item.

\section{À GUISA DE CONCLUSÃO}

Destacamos ser bastante comum o fato de itens componentes de testes possuírem algum tipo de viés, dentre os quais o DIF. Como enfatizamos anteriormente, o DIF ocasiona sérios problemas às avaliações educacionais. Trata-se de um fator de injustiça para alguns grupos de respondentes, pois os alunos que possuem o mesmo grau de aprendizagem e que provêm de distintos grupos demográficos têm distintas probabilidades de acertar um mesmo item. Portanto, devemos reconhecer a relevância das investigações acerca do DIF, já que podem proporcionar maior eqüidade aos processos de avaliação educacional, através da identificação e não-utilização de itens com algum tipo de DIF. Por fim, devemos enfatizar a opinião de Andriola (2002):

(...) los estudios para detectar el DIF - mezclando procedimientos cualitativos y cuantitativos - deben ser efectuados inmediatamente después de la elaboración de los ítems que compondrán dichos instrumentos de medida. (p. 554)

Além disso, é preciso fazer menção ao fato de que a área de investigação do DIF, no âmbito educativo e psicológico, é recente, necessitando de boas hipóteses, fundamentadas em teorias científicas, que tentem "abrir novas perspectivas" aos estudos do DIF (Cole, 1993; Hambleton, 1997; Roznowski, Reith, 1999; Scheuneman, Gerritz, 1990). Como declara Bond (1993): de modo geral, teorias acerca do porquê itens funcionam de forma diferente para certos grupos podem ser descritas como primitivas (p. 278).

Schmitt, Holland e Dorans (1993) acreditam que a área que investiga o DIF não tem progredido no grau desejado em virtude de três fatores:

- porque as investigações acerca do DIF são relativamente recentes e, atualmente, a ênfase está no desenvolvimento de 
métodos estatísticos para a sua identificação. Por exemplo, as modernas técnicas para a detecção do funcionamento diferencial das alternativas (DAF) têm o mesmo objetivo das técnicas DIF, isto é, compreender as causas da escolha diferenciada das alternativas de um item por sujeitos que têm o mesmo nível de habilidade, mas fazem parte de distintos grupos demográficos (Thissen, Steinberg, Wainer, 1993; Thissen, Steinberg, Fitzpatrick, 1989);

- porque a identificação do DIF e os fatores a ele relacionados necessitam de boas teorias sobre a dificuldade diferencial dos itens, em um campo no qual as teorias sobre os processos cognitivos presentes na resolução dos itens não se encontram, todavia, minimamente avançadas;

- porque a identificação e descrição dos citados processos cognitivos são muito complexas, uma vez que intervêm múltiplos fatores. Além disso, é um campo de investigação que exige o trabalho multidisciplinar de psicólogos, pedagogos e matemáticos; algo extremamente difícil no estágio atual de desenvolvimento investigativo brasileiro.

Devemos dizer que o processo de criação de boas hipóteses explicativas do DIF deverá, logicamente, ser árduo, difícil e frustrante. As hipóteses deverão sofrer corroborações e rejeições, algo bastante comum à atividade científica (Wilson, 1999). Assim, verificamos que, apesar de existir grande variedade de métodos para investigar o DIF, os mesmos sofrem limitações. Autores mais críticos aconselham complementar as análises estatísticas, obtidas pelo uso de mais de um procedimento de detecção do DIF, com a opinião de especialistas na área e, assim, aumentar a validade dos resultados.

Além disso, devemos reconhecer que a presença do DIF em itens de instrumentos de medida psicológica e pedagógica é um grave problema que atenta contra o pressuposto da padronização ou uniformização das condições de avaliação. É uma fonte de injustiça, já que produz falta de eqüidade nos processos avaliativos; permite aos sujeitos, com mesmo grau na variável latente ou construto medido pelo item, obter melhores resultados, visto que esses têm maior probabilidades de acertá-lo.

Desse modo, caberá aos responsáveis pela construção, administração e comercialização de testes pedagógicos e psicológicos, verificar a presença de itens com DIF em seus instrumentos, uma vez que a sua existência é um fator de invalidação dos resultados. Também os psicometristas que começam a organizar bancos de itens necessitam 
verificar a presença de DIF e, assim, evitar utilizá-los em processos avaliativos (Andriola, 1998).

Para finalizar, mencionaremos célebre frase latina que é muito sugestiva e sintetiza, na nossa opinião, a importância dos estudos acerca do DIF no âmbito da avaliação psicológica e educacional: satius est initio mederi, quam fini (é melhor remediar no princípio do que no fim).

\section{REFERÊNCIAS BIBLIOGRÁFICAS}

ALLEN, N. L.; WAINER, H. Nonresponse in Declared Ethnicity and the Identification of Differential Functioning Items. Technical Reports, n. 89-89. New Jersey: Educational Testing Service (ETS), 1989.

ANASTASI, A. Psychological Testing. New York: McMillan, 1988.

ANDRIOLA, W. B. Detección del funcionamiento diferencial del ítem (DIF) en tests de rendimiento. Aportaciones teóricas y metodológicas. Madrid, 2002. Tese (dout.) Universidad Complutense de Madrid.

Funcionamento Diferencial do Item (DIF): estudo com analogias para medir o raciocínio verbal. Psicologia: Reflexão e Crítica, v. 13, n. 3, p. 473-481, 2000.

Utilização da teoria de resposta ao item (TRI) para a organização de um banco de itens destinados à avaliação do raciocínio verbal. Psicologia: Reflexão e Crítica, v. 11, p. 295-308, 1998.

Avaliação do raciocínio verbal em estudantes do 2o grau. Estudos de Psicologia, v. 2, n. 2, p. 277-285, 1997.

ANDRIOLA, W. B.; PASQUALI, L. A Construção de um Teste de Raciocínio Verbal (RV). Psicologia: Reflexão e Crítica, v. 8, n. 1, p. 51-72, 1995.

ANGOFF, W. H. Perspectives on Differential Item Functioning (p. 3-23). In: HOLLAND, P. W.; WAINER, H. (eds.). Differential Item Functioning. New Jersey: Lawrence Erlbaum Associates, 1993. 
BOCK, R. D. Different DIF's: comment on the Papers Read by Neil Dorans and David Thissen (p. 115-122). In: HOLLAND, P. W.; WAINER, H. (eds.). Differential Item Functioning. New Jersey: Lawrence Erlbaum Associates, 1993.

CAMILLI, G.; SHEPARD, L. A. MMSS. Methods for Identifying Biased Test Items. California: SAGE Publications, 1994.

CLAUSER, B. E.; NUNGESTER, R. J.; SWAMINATHAN, H. Improving the matching for DIF analysis by conditioning on both test score and an educational background variable. Journal of Educational Measurement, v. 33, n. 4, p. 453-464, 1996.

COHEN, A. S.; KIM, S.; BAKER, F. B. Detection of differential item functioning in the graded response model. Applied Psychological Measurement, v. 17, n. 4, p. 335-350, 1993.

COLE, N. S. History and Development of DIF (p. 25-29). In: HOLLAND, P. W.; WAINER, H. (eds.). Differential Item Functioning. New Jersey: Lawrence Erlbaum Associates, 1993.

COLE, N. S.; MOSS, P. A. Bias in test use (p. 201-219). In: LINN, R. L. (ed.) Educational Measurement. New York: McMillan, 1989.

DOUGLAS, J. A.; ROUSSOS, L. A.; STOUT, W. Item-Bundle DIF hypothesis testing: identifying suspect bundles and assessing their differential functioning. Journal of Educational Measurement, v. 33, n. 4, p. 465-484, 1996.

DOWNING, S. M.; HALADYNA, T. M. Test item development: Validity evidence from quality assurance procedures. Applied Measurement in Education, v. 10, n. 1, p.61-82, 1997.

ERCIKAN, K. Translation effects in international assessments. International Journal of Educational Research, n. 29, p. 543-553, 1998.

HAMBLETON, R. K. Perspectivas futuras y aplicaciones (p. 203-213). In: MUÑIZ, J. Introducción a la Teoría de Respuesta a los Ítems. Madrid: Ediciones Psicología Pirámide, 1997.

Item response theory: introduction and bibliography. Psicothema, v. 2, n. 1, p.97-107, 1990. 
HAMBLETON, R. K. Principles and selected applications of item response theory (p. 147-200). In: LINN, R. L. (ed.). Educational Measurement. New York: McMillan, 1989.

HAMBLETON, R. K.; SWAMINATHAN, H.; ROGERS, H. J. Fundamentals of Item Response Theory. North Caroline: Sage Publications, 1991.

HARTLE, T. W.; BATTAGLIA, P. A. The Federal Role in standardized testing (p. 291-311). In: BENNETT, R. E.; WARD, W. C. (org.), Construction versus Multiple Choice Items in Cognitive Measurement. New Jersey: Lawrence Erlbaum Associates, 1993.

HIDALGO MONTESINOS, M. D.; LÓPEZ PINA, J. A.; SÁNCHEZ MECA, J. Error tipo I y potencia de las pruebas chi-cuadrado en el estudio del funcionamiento diferencial de los ítems. Revista de Investigación Educacional, v. 15, n. 1, p. 149-168, 1997.

LINN, R. L.; HARNISCH, D. L. Interactions between item content and group membership on achievement test items. Journal of Educational Measurement, n. 18, p. 109-118, 1981.

LORD, F. M. Applications of Item Response Theory to Practical Testing Problems. New Jersey: Lawrence Erlbaum Associates, 1980.

MARTÍNEZ ARIAS, R. Psicometría: Teoría de los Tests Psicológicos y Educativos. Madrid: Ediciones Síntesis, 1997.

MAZOR, K. H.; HAMBLETON, R. K.; CLAUSER, B. E. Multidimensional DIF analyses: the effects of matching on unidimensional subtest scores. Applied Psychological Measurement, v. 22, n. 4, p. 357-367, 1998.

MELLENBERGH, G. J. Item bias and item response theory. International Journal of Educational Research, v. 13, n. 2, p. 127-143, 1989.

MISLEVY, R. J. Test theory reconceived. Journal of Educational Measurement, v. 33, n. 4, p. 379-416, 1996.

MUÑIZ, J. Introducción a la Teoría de Respuesta a los Ítems. Madrid: Ediciones Psicología Pirámide, 1997.

Teoría Clásica de los Tests. Madrid: Ediciones Pirámide S.A., 1994. 
OSHIMA, T. C.; RAJU, N. S.; FLOWERS, C. P.; SLINDE, J. A. Differential Bundle functioning using the DFIT framework: procedures for identifying possible sources of differential functioning. Applied Measurement in Educational, v. 11, n. 4, p. 353-369, 1998.

OSTERLIND, S. J. Test item bias. Beverly Hills: Sage Publications, 1979.

Constructing test items. Boston: Kluwer Publications, 1989.

PASQUALI, L. Psicometria: Teoria dos Testes Psicológicos. Brasília: Prática Gráfica, 2000.

REE, M. J. Foreword: Differential Item Functioning (DIF): a perspective from the Air Force Human Resources Laboratory (p. xi-xii). In: HOLLAND, P. W.; WAINER, H. (eds.). Differential Item Functioning. New Jersey: Lawrence Erlbaum Associates, 1993.

ROZNOWSKI, M.; REITH, J. Examining the measurement quality of tests containing differentially functioning items: do biased items result in poor measurement? Educational and Psychological Measurement, v. 52, n. 2, p. 248$269,1999$.

SCHEUNEMAN, J. D.; GERRITZ, K. Using differential item functioning procedures to explore sources of item difficulty and group performance characteristics. Journal of Educational Measurement, v. 27, n. 2, p.109-131, 1990.

SCHMITT, A. P.; HOLLAND, P. W.; DORANS, N. J. Evaluating Hyphoteses about Differential Item Functioning (p. 281-319). In: HOLLAND, P. W.; WAINER, H. (eds.). Differential Item Functioning. New Jersey: Lawrence Erlbaum Associates, 1993.

THISSEN, D.; STEINBERG, L.; FITZPATRICK, A. R. Multiple-choice models: the distractors are also part of the item. Journal of Educational Measurement, v. 26, n. 2, p.161-176, 1989.

THISSEN, D.; STEINBERG, L.; WAINER, H. Detection of Differential Item Functioning using the Parameters of Item Response Models (p. 67-113). In: HOLLAND, P. W.; WAINER, H. (eds.). Differential Item Functioning. New Jersey: Lawrence Erlbaum Associates, 1993. 
WHITMORE, M. L.; SHUMACKER, R. E. A Comparison of logistic regression and analysis de variance differential item functioning detection methods. Educational and Psychological Measurement, v. 59, n. 6, p. 910-927, 1999.

WILSON, E. O. Consilience: la Unidad del Conocimiento. Barcelona: Ediciones Galaxia Gutemberg, 1999.

Recebido em: abril 2006

Aprovado para publicação em: agosto 2006 